Ann. Zootech., I974, $23(4), 435-444$.

\title{
EFFETS DE LA SUPPRESSION DE LA TRAITE DU DIMANCHE SOIR SUR LES BREBIS DE RACE PREALPES DU SUD
}

\author{
J. LABUSSIÈRE, J.-F. COMBAUD et P. PETREQUIN \\ avec la collaboration technique de P. Chesne et M. Oechli \\ Laboratoire de Recherches sur la Physiologie et la Technologie de la Traite, \\ Centre de Recherches de Rennes, I. N.R.A., \\ 35042 Rennes Cedex
}

\begin{abstract}
RÉSUMÉ
Cinquante agnelles Préalpes du Sud sont traites 2 fois par jour, à 6 heures et I 6 heures sauf le dimanche soir (le Iait s'accumule donc dans la mamelle pendant 24 heures).

Par rapport au lot témoin (qui comprend également 50 agnelles) cette suppression entraîne, sur les rio premiers jours de lactation une réduction de 25,6 p. I0o, 22,6 p. Ioo et I 7,5 p. Ioo respectivement pour la production du lait, des matières grasses et des matières azotées (fig. 2 et tabl. I).

Le tracé en "dent de scie " des modifications journalières de ces 3 paramètres se situe alors à un niveau très inférieur à celui des animaux traits normalement 2 fois par jour et l'activité secrétoire des cellules mammaires est très perturbée jusqu'au mercredi soir (fig. 3, 4 et 5).

Nous n'avons pas constaté, par contre, de modifications de l'état sanitaire du pis (tabl. 3 ).
\end{abstract}

\section{I. - INTRODUCTION}

Les effets de la suppression de la traite du dimanche soir ont surtout été étudiés chez les bovins ; la réduction de production laitière varie entre 4 et $8 \mathrm{p}$. Ioo suivant les intervalles entre traites choisis par les différents auteurs (voir la bibliographie dans LABUSSIÈRE et CorNDET Ig68). La composition du lait est généralement perturbée jusqu'au mercredi matin, mais l'état sanitaire du pis n'est pas affecté.

La seule expérimentation que nous connaissions chez les ovins est celle de CASU et LABUssière (I972) sur la Brebis Sarde qui possède une citerne dont les possibilités de stockage sont très importantes. 
Malgré ces caractéristiques particulièrement intéressantes, la " perte " observée par ces auteurs (I 2,8 p. I00) est légèrement supérieure à celle mentionnée précédemment dans l'espèce bovine. Ces conclusions portant sur un effectif limité mériteraient d'être confirmées, mais on est en droit de se demander si les résultats d'une telle pratique ne seraient pas encore plus défavorables chez la Brebis Préalpes du Sud, puisque dans cette race :

- le volume de la citerne est beaucoup plus réduit;

- un nombre élevé d'animaux retiennent naturellement leur lait dans les parties supérieures de la mamelle (LABUSSIÈRE I966) ce qui peut freiner fortement l'activité des cellules sécrétoires.

\section{II. — MATÉRIEI, ETT MÉTHODES}

\section{A. - Caractéristiques du troupeau et constitution de 2 lots expérimentaux}

Les Ioo agnelles Préalpes $d u$ Sud sur lesquelles porte cette expérience, sont saillies par groupe de ro, entre le 20 avril et le 2 I mai $197^{\circ}$, après incluction des chaleurs à contre saison selon la technique proposée par Thimonien et al. en 1968 (éponges imprégnées de $30 \mathrm{mg}$ d'acétate de Fluorogestone maintenues pendant I $_{4}$ jours dans le vagin et injection intramusculaire de foo UI de PMSG le jour du retrait).

Les mises bas s'échelonnent donc entre le 9 septembre et le i 7 octobre 1970. Après une courte phase d'allaitement (en moyenne 2 jours) les brebis sont séparées de leur jeune et au fur et à mesure des agnelages sont affectées alternativement :

- au lot témoin (T) qui est trait 2 fois par jour à 6 heures et à 16 heures ;

- au lot suppression (S) qui subit le même traitement à l'exception du dimanche soir où la traite est toujours supprimée et ceci dès le début de la lactation.

\section{B. - Technique de traite}

La salle de traite de type casse $2 \times$ I 2 (Bosc, I 963) est équipée d'une machine Alfa Laval dont les caractéristiques principales sont les suivantes :

- vide $33 \mathrm{~cm} / \mathrm{Hg}$;

— vitesse de pulsation $180 /$ minute ;

- rapport succion massage 50 p. Ioo (normes F. I. L. 56 de I970) ;

- I 2 faisceaux trayeurs équipés de gobelets no 26895 (poids I $44 \mathrm{~g}$, intérieur $33 \mathrm{~mm}$ hauteur I IO $\mathrm{mm}$ ) et des manchons $\mathrm{n}^{\circ} 25200$ ( $\varnothing 19 \mathrm{~mm}$ ).

La griffe est mise en place sans " préparation " préalable de la mamelle avec un linge chaud, Lorsque le lait cesse de couler, le trayeur pratique un égouttage à la machine, dépose les gobelets puis repose une seconde fois. Cette méthode préconiséc par Bosc, Flamand et RICordeaU (1967) permet d'extraire les dernières fraction de lait qui sont habituellement recueillies lors des opérations d'égouttage manuel.

\section{C. - Contrôles expérimentaux}

La figure I indique que les 7 semaines de contrôle débutent alternativement tous les I 7 ou I 8 jours puisque pour des raisons d'organisation du travail les mesures individuelles sont effectuées à chaque traite :

- Pour les matières grasses (gerber) et les matières azotées (noir amido) uniquement au cours des semaines paires $\left(S_{2}, S_{4}, S_{6}\right)$ du vendredi matin au jeudi soir inclus.

- Pour la production laitière à la fois au cours des semaines paires (même limite que ci-dessus) et impaires $\left(S_{1}, S_{3}, S_{5}\right.$ et $\left.S_{7}\right)$ du mardi matin au lundi soir inclus. La quantité de lait est appréciée par pesée avec une précision voisine de 5 grammes. 
Les échantillons prélevés le mardi après-midi des semaines $S_{2}, S_{4}$ et $S_{6}$ sont également utilisées pour vérifier l'état sanitaire de la mamelle. La méthode choisie est le California Mastitis Test (CMT) qui permet d'apprécicr sommairement le nombre de leucocytes du lait.

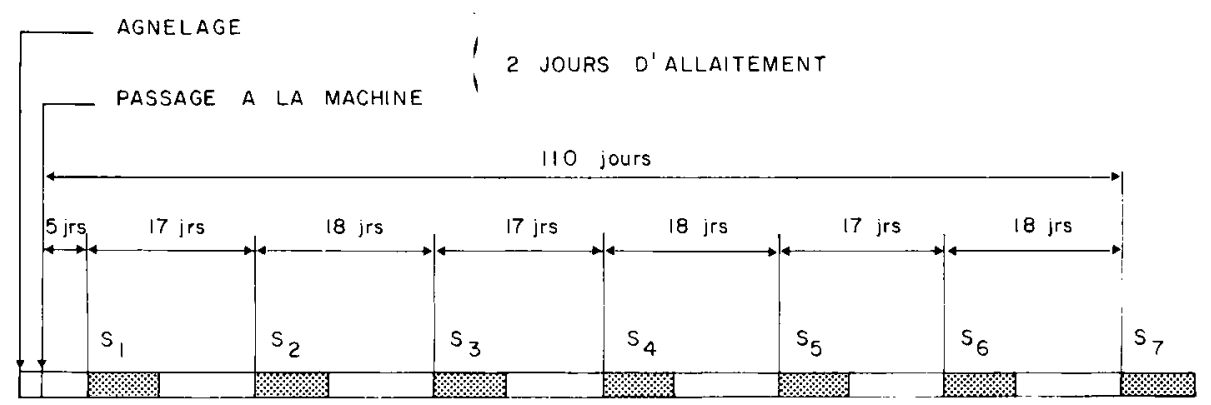

FIG. I. - Organisation des différents contrôles au cours de l'expérience

contrôle de production laitière : semaines $S_{1} S_{2} S_{3} S_{4} S_{5} S_{6} S_{7}$ analyses des matières grasses et azotées $S_{2} S_{4} S_{6}$

test CMT : le mardi soir de $S_{2} S_{4} S_{6}$

\section{III. — RÉSULTATS}

\section{A. - Bilan des 110 jours expérimentaux}

La figure 2 fait apparaître un écart important entre les courbes de lactation du lot témoin et du lot suppression. L’intervalle de confiance calculé pour chaque point au seuil de 5 p. Ioo, indique que les 2 tracés sont significativement différents.

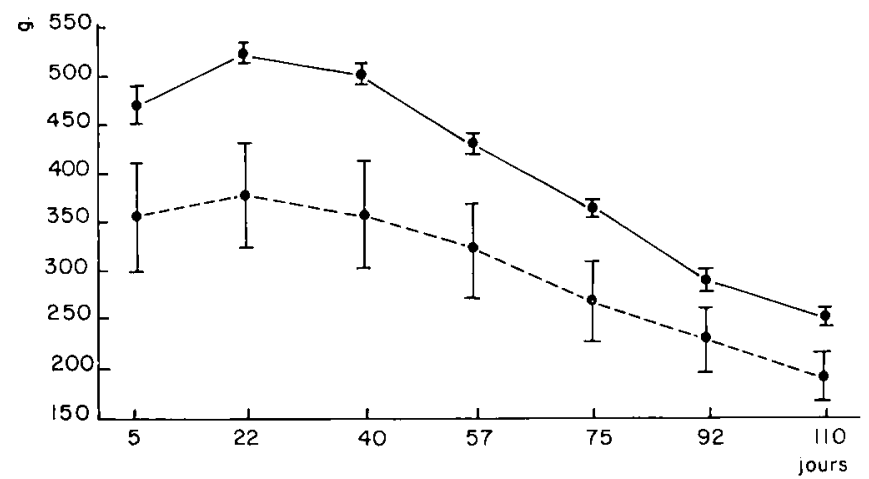

FIG. 2. - Effets de la suppression de la traite du dimanche soir sur les courbes de lactation des lots:

$$
\text { ....... Suppression }
$$

Les écarts par rapport aux valeurs moyennes représentent l'intervalle de confiance

L,e tableau I, qui exprime les rendements obtenus en I Io jouts et la composition moyenne du lait recueilli, prouve qu'il en est de même pour l'ensemble des critères que nous avons contrôlés. 
C'est ainsi que les brebis qui ne sont pas traites le dimanche soir fournissent respectivement 74,4I p. I00, 77,38 p. Ioo et $82,46 \mathrm{p}$. Ioo du lait, des matières grasses et des matières azotées produites par les animaux témoins. On remarquera que si la perte des 2 principaux constituants est un peu plus faible que celle du lait proprement dit, ceci résulte de l'augmentation significative du taux butyreux et du taux azoté dans le lot témoin.

\section{TABLEAU I}

Effots dc la suppression de la traite du dimanche soir sur la production du lait, des matières grasses et des matières azotées

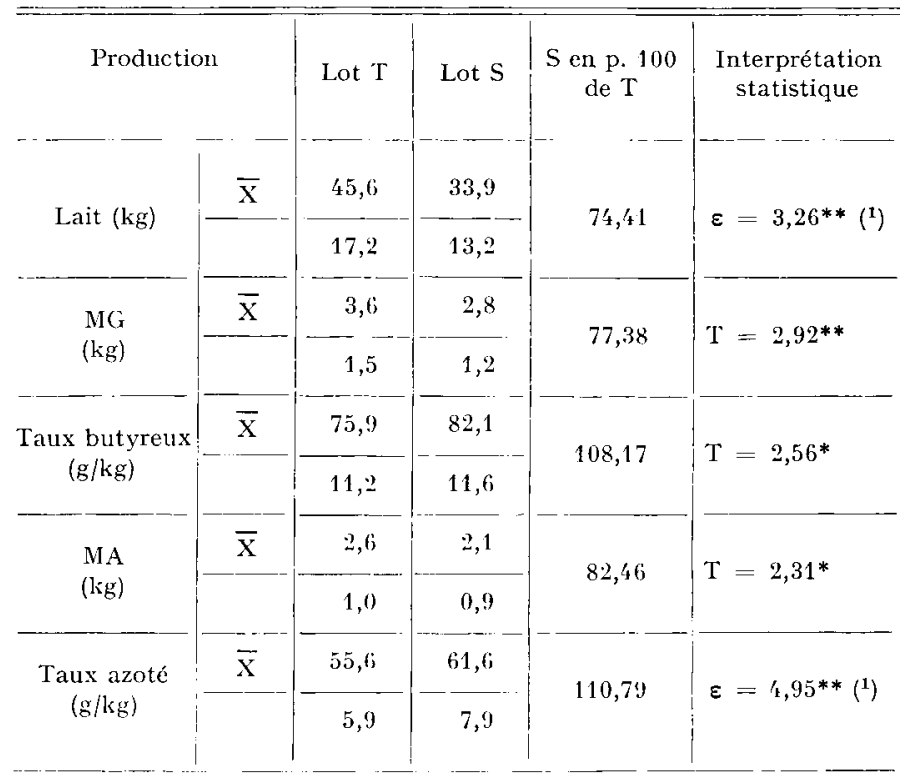

Lot $\mathrm{T}=$ lot témoin (50 animaux)

Lot $\mathrm{S}=$ lot suppression (50 animaux)

(1) Test non paramétrique de Mann-Whitney, Wilcoxon-White

* Différence significative au niveau 5 p. 100

** Différence significative au niveau 1 p. 100

\section{B. - Modifications journalières de la production et de la composition du lait}

Les résultats que nous allons brièvement commenter ci-dessous sont présentés au tableau 2 et aux figures 3,4 et 5 . Le lait (fig. $3 a$ ), les matières grasses (fig. $4 a$ ) et les matières azotées (fig. $5 a$ ) fournis par les brebis non traites le dimanche soir, évoluent selon un tracé en "dent de scie ", qui, à l'exception du lundi matin, se situe à un niveau inférieur à celui des animaux traits normalement 2 fois par jour. On assiste par contre à une situation inverse pour le taux butyreux (fig. $4 b$ ) et le taux azoté (fig. 5 b) ${ }^{1}$ ).

(1) Le nombre d'analyses au noir amido étant probablement insuffisant, il subsiste des irrégularités sur le tracé du taux azoté qui ne nous permettent pas de tirer des conclusions valables pour ce critère. 


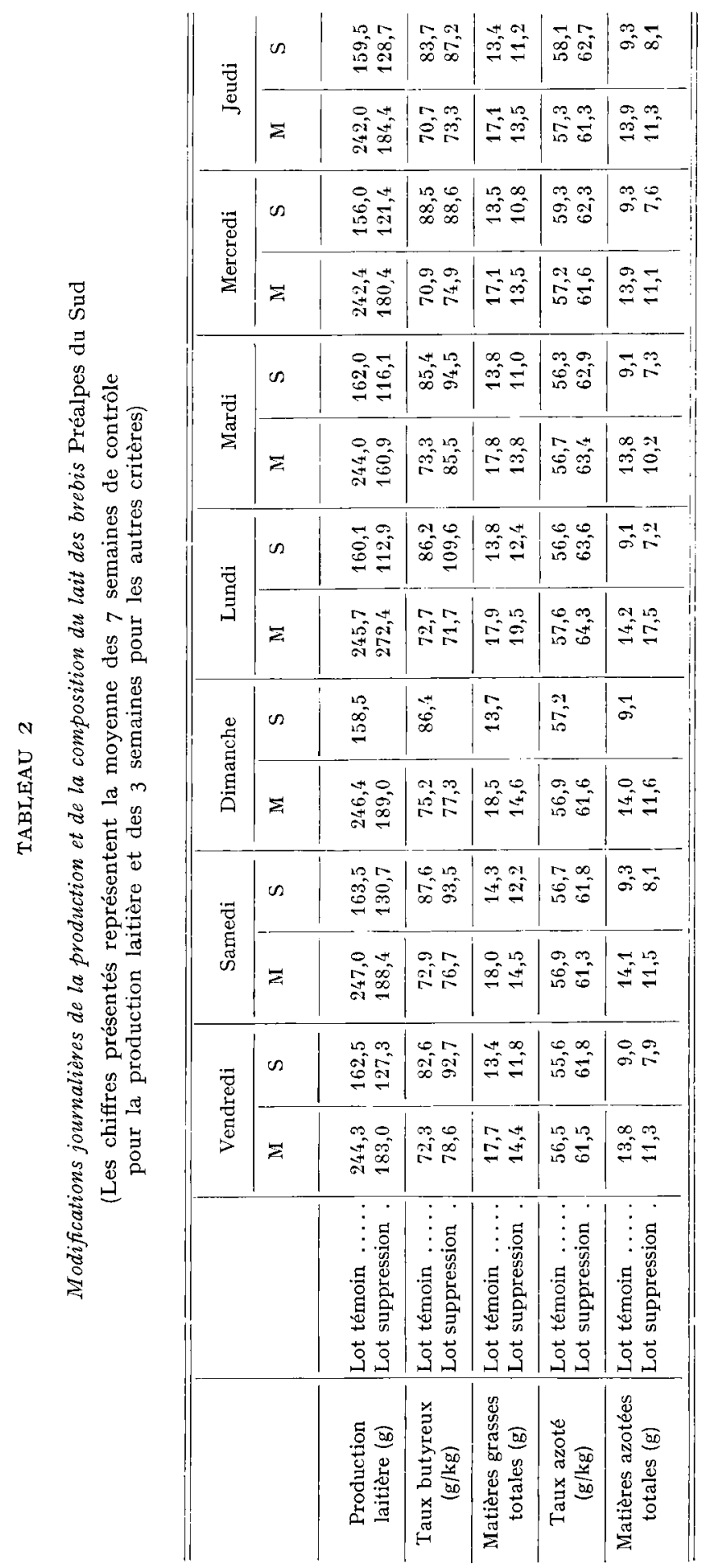



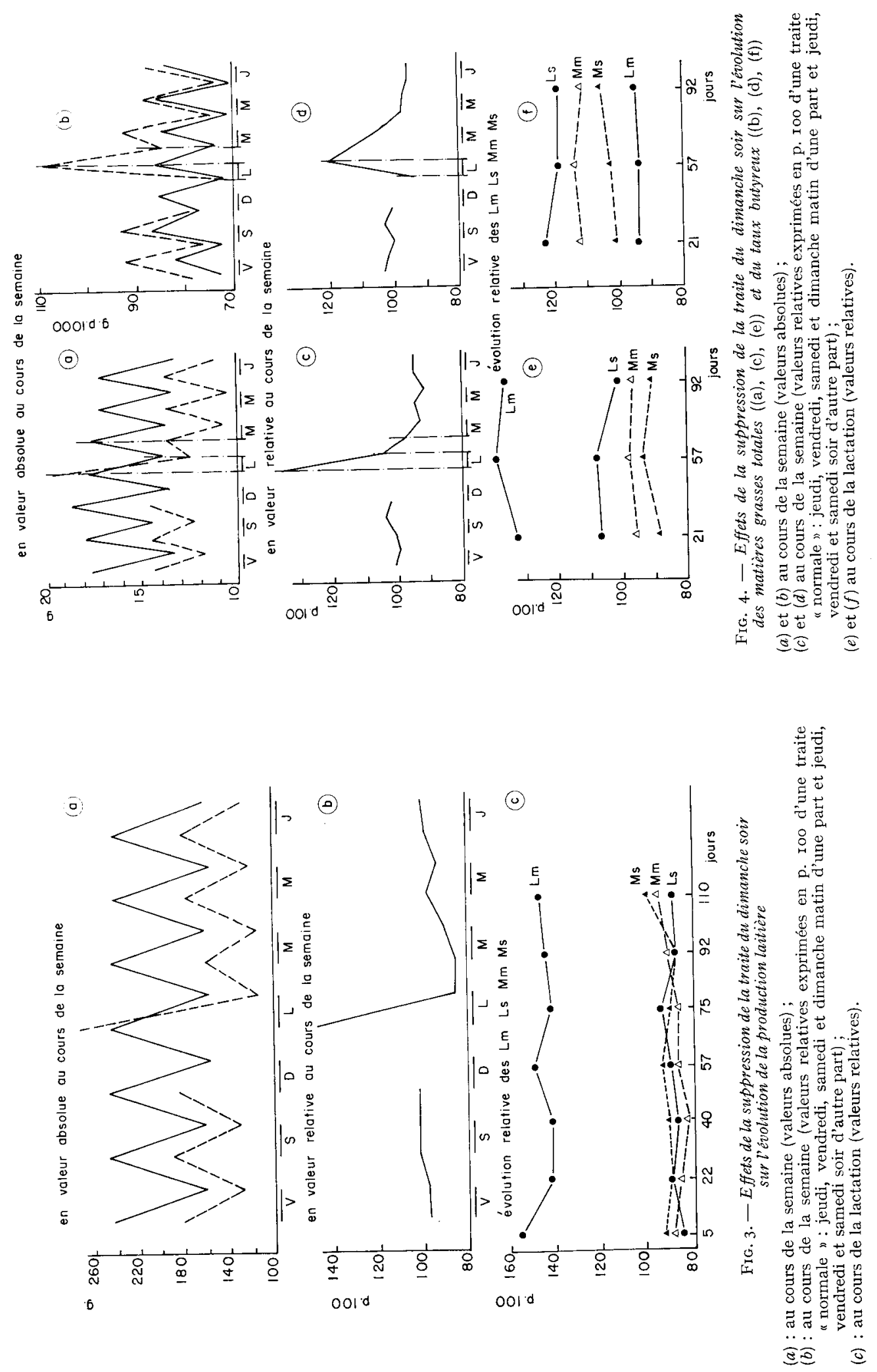
Si l'on se referre au lot témoin il n'y a donc jamais " retour à la normale " après l'omission de la traite du dimanche soir et c'est la raison pour laquelle nous avons également choisi d'illustrer les perturbations qui en résultent au sein même du lot " suppression ", en exprimant chaque résultat en valeur relative par rapport à la moyenne des jeudi, vendredi, samedi et dimanche matin d'une part (IOo p. IOO) et des jeudi, vendredi et samedi soir d'autre part (roo p. Ioo).

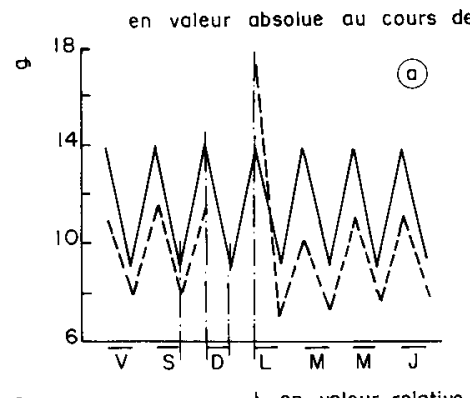

la semoine
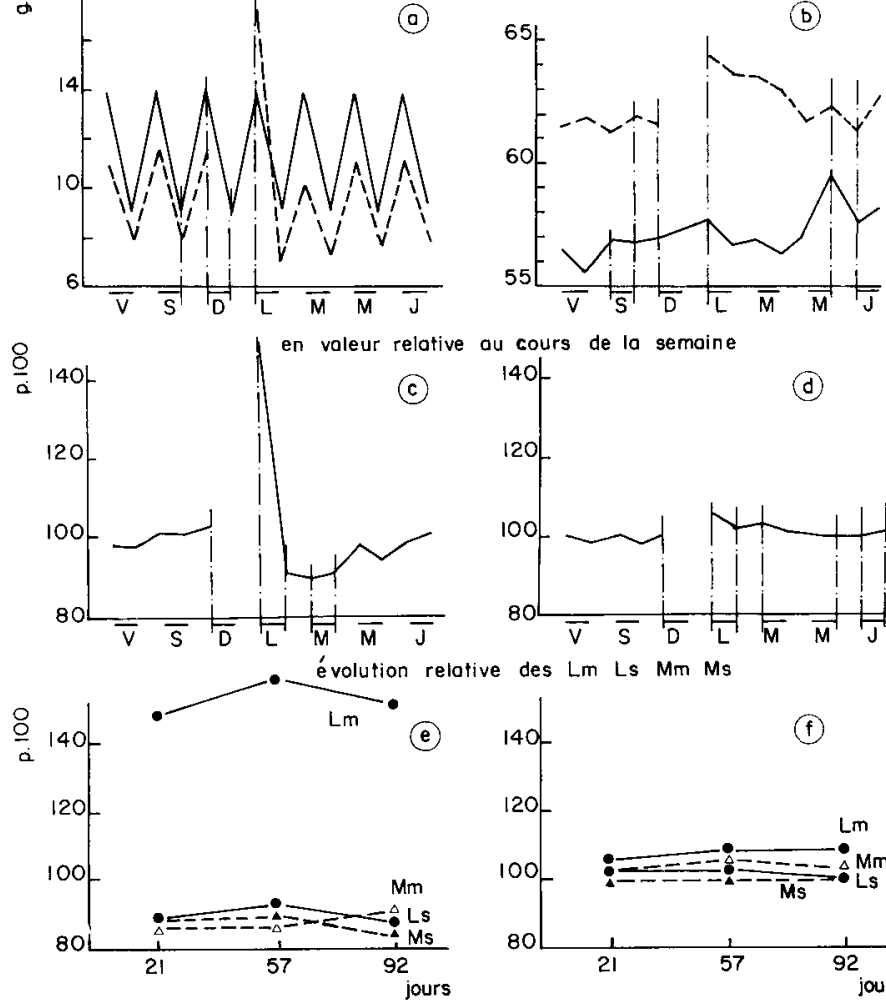

(d)

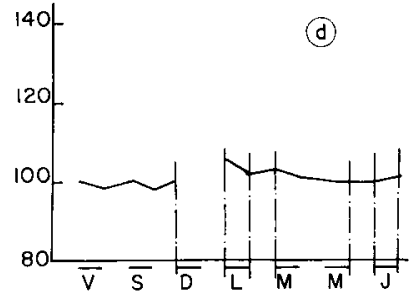

(9)

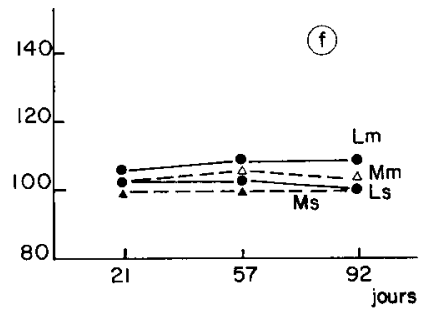

FIG. 5. - Effets de la suppression de la traite du dimanche soir

sur l'évolution des matières azotées totales $((\mathrm{a}),(\mathrm{c}),(\mathrm{e}))$ et du taux azoté ((b), (d), (f)\}

(a) et (b) au cours de la semaine (valeurs absolues);

(c) et (d) au cours de la semaine (valeurs relatives exprimées en p. Ioo d'une traite "normale»: jeudi,

vendredi, samedi et dimanche matin d'une part et jeudi, vendredi et samedi d'autre part);

(e) et (f) au cours de la lactation (valeurs relatives).

Les modifications les plus importantes sont perçues :

a) Le lundi matin pour le lait (fig. $3 b$ ) les matières grasses (fig. $4 c$ ) les matières azotées (fig. $5 \mathrm{c}$ ) puisque les quantités obtenues alors représentent respectivement I46, I37 et I5O p. Ioo de ce qui devrait habituellement être recueilli un matin " ordinaire ». On remarquera aux figures $3 c, 4 e$ et $5 e$ que ces pourcentages restent pratiquement les mêmes tout au long des I 2 jours de lactation étudiés.

b) Le lundi soir pour le taux butyreux qui s'élève en moyenne à IIo $\mathrm{g} / \mathrm{kg}$ (fig. 4 b). Ceci correspond à une valeur relative de $\mathbf{2} 20$ p. Ioo (fig. $4 d$ ) qui, elle aussi, 
a tendance à se maintenir identique jusqu'à la $3^{\mathrm{e}}$ et dernière semaine de contrôle

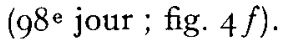

La sécrétion se régularise ensuite peu à peu le mardi et le mercredi (voir surtout la fig. $3 b$ ) et il semble préférable que le contrôleur laitier attende le jeudi matin pour intervenir dans les exploitations ou la suppression de la traite du dimanche soir serait pratiquée.

\section{C. - Contrôle de l'état sanitaire de la mamelle.}

L'omission de la traite du dimanche soir ne semble pas influencer défavorablement l'état sanitaire de la mamelle des brebis Préalpes du Sud.

Nous n'avons en effet constaté aucun cas de mammite clinique et sur les 300 échantillons de lait examinés, le California Mastitis Test s'est avéré très légèrement positif, seulement 2 fois dans le lot suppression et une fois dans le lot témoin (tabl. 3).

TABLEAU 3

Effet de la suppression de la traite du dimanche soir sur la notation du California Mastitis Test (CMT)

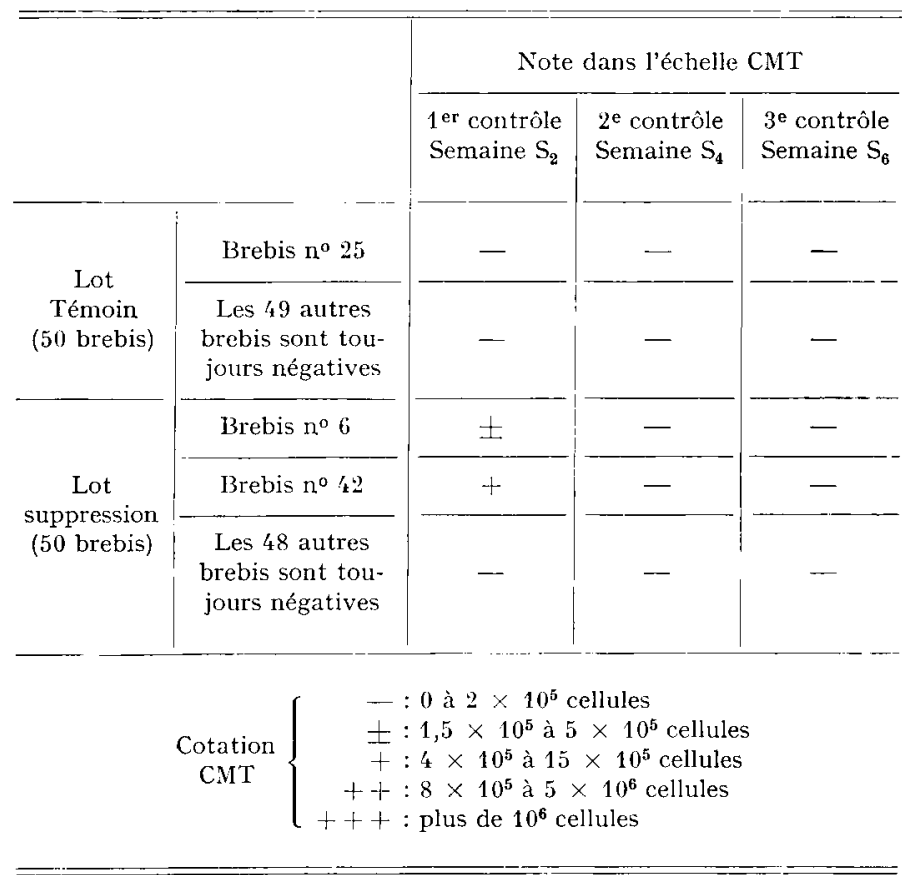

IV. - DISCUSSION E'T CONCLUSION

Bien que l'état sanitaire de la mamelle soit respecté après la suppression de la traite du dimanche soir, il ne semble pas économiquement possible d'adopter une telle technique avec des agnelles Préalpes $d u$ Sud compte tenu de la réduction importante 
de production que nous avons enregistrée non seulement pour le lait $(25,6$ p. Ioo) mais aussi pour les matières grasses (22,6 p. IOO) et les matières azotées (I7,5 p. IOO).

Ces chiffres sont nettement supérieurs à ceux mentionnés par CASU et LABUsSIÈRE (I972) chez des brebis adultes de race Sarde puisque les pertes pour les 3 critères précédents s'élevaient respectivement à $\mathrm{I} 2,8$ p. IOO, I4,O p. IOO et 9,9 p. IOO).

Il se confirme donc que l'accumulation du lait pendant 24 heures dans le pis des animaux Préalpes, entraîne une diminution très sensible de l'activité secrétoire des cellules mammaires. Rappelons en effet qu'avec des brebis de même race, LABusSière, Combaud et Petrequin (I974) obtiennent un gain de 5I,3 p. Ioo en passant de une à 2 traites par jour.

Les perturbations sensibles de la composition du lait pendant les 2 ou 3 premiers jours qui suivent la suppression de la traite du dimanche soir, doivent être également pris en considération par :

- les industriels laitiers, car la qualité fromagère risque d'être différente d'un jour à l'autre ;

- les contrôleurs laitiers, qui ne pourront intervenir avant le jeudi dans les fermes qui souhaiteraient adopter cette technique.

L'expérience précédente portant sur des agnelles, il est néanmoins probable que les résultats auraient été un peu plus favorables avec des adultes :

- qui supportent très certainement des évacuations plus espacées puisque le réflexe d'éjection du lait apparaît alors plus fréquemment (LABUSSIÈRE et RICORDEAU I970) ;

- qui présentent une capacité de stockage citernal plus importante bien que très inférieure à celle de la Brebis Sarde.

Cette hypothèse mérite donc d'être vérifiée avant de déconseiller définitivement aux éleveurs la pratique d'une telle suppression, non seulement dans la race Préalpes mais aussi dans la race Lacaune qui lui est très semblable et qui est beaucoup plus utilisée pour la spéculation laitière.

Reçu pour publication en avril 1974.

\section{REMERCIEMEN'TS}

Nous tenons à remercier très vivement le personnel de la ferme expérimentale de Brouessy sans l'aide duquel ce travail n'aurait pu être réalisé.

\section{SUMMARY}

EFFECTS OF THE SUPPRESSION OF SUNDAY EVENING MILKING

OF EWES OF THE PRÉALPES DU SUD BREED

Fifty ewe lambs of the Préalpes $d u$ Sud breed were milked twice a day, at 6 a.m. and 4 p.m., except on Sunday evenings, resulting in an accumulation of milk in the mammary gland for 24 hours. 
As compared to the control group (also including 50 ewe lambs), this suppression entailed, for the first I Io days of lactation, a reduction of $25.6,22.6$ and $17.5 \mathrm{p}$. Ioo, respectively, for milk yield, fat and crude protcin (fig. 2 and table $I$ ).

The "serrated " curve corresponding to the daily changes in these 3 parameters is situated at a level far below that of the animals milked normally twice a day, and the secretory activity of the mammary cells was much affected until Wednesday evening (fig. 3, 4 and 5).

On the other hand, no modifications in the sanitary state of the udder were noticed (table 3 ).

\section{RÉFÉRENCES BIBLIOGRAPHIQUESS}

Bosc J., 1963. Problèmes de l'organisation du travail dans un troupeau de brebis laitières. Adaptation de la méthode arête de poisson à la traite mécanique des brebis. Compte-rendu à l'A.F. Z. 3-39.

Bosc J., Flamand J. C., Ricordeau G., I967. Traite à la machine des brebis. Suppression de l'égouttage manuel et remplacement par un égouttage machine. Ann. Zootech., 16, I9t-202.

Casu S., Labussière J., I972. Premiers résultats concernant la suppression d'une ou plusieurs traites par semaine chez la Brebis Sarde. Ann. Zootech., 21, 223-232.

LABUSsière J., I966. Relation entre le niveau de production laitière des brebis et leur aptitude à la traite. $X V I I^{\mathrm{e}}$ Congrès International de Laiterie (Munich) Section AI, 43-5I.

Labussière J., CoIndet J., i968. Effets de la suppression de la traite du dimanche soir chez les bovins de race Française Frisonne Pie noire. Ann. Zootech., 17, 231-244.

Labussit̀re J., Ricordeau S., I970. Aptitude à la traite mécanique des brebis de race Préalpes du Sud et croisées Frisons $\times$ Préalpes. Étude à différents stades de la lactation. Ann. Zootech., 19, I59-I9o.

Labussikere J., Combaud J. F., Petrequin P., I974. Infiuence de la fréquence des traites et des tétées sur la production laitière des brebis Préalpes du Sud. Ann. Zootech., 23, 445-457.

Thimonier J., Mauleon P., Cognie Y., Ortavant R., ig68. Déclenchement de l'oestrus et obtention de la gestation pendant l'anoestrus post-partum chez les brebis à l'aide d'éponges vaginales imprégnées d'Acétate de Fluorogestone. Ann. Zootech., 17, 257-273. 